\title{
GAMMAGRAFÍA CON OCTREÓTIDO EN LA ORBITOPATÍA DISTIROIDEA
}

\section{OCTREOTIDE SCINTIGRAPHY IN THYROID ORBITOPATHY}

\author{
AGUIRRE-BALSALOBRE $\mathrm{F}^{1}$, MENGUAL-VERDÚ $\mathrm{E}^{2}$, MUÑOZ-ACOSTA JM ${ }^{1}$, \\ MARTÍNEZ-CABALLERO A ${ }^{2}$, CABALLERO-CARPENA O ${ }^{2}$, HUESO-ABACENS JR ${ }^{2}$
}

\section{RESUMEN}

Introducción: La enfermedad de Graves es una enfermedad de base autoinmune en la cual existe una infiltración por linfocitos activados en el tejido retroorbitario. Estos linfocitos activados se caracterizan por aumentar la expresión de receptores de somatostatina en su superficie que median la respuesta inflamatoria.

Objetivo: Valorar la efectividad de la gammagrafía con ${ }^{111}$ In-octreótido en la identificación de actividad orbitaria en la Enfermedad de Graves y valorar qué pacientes son subsidiarios de recibir tratamiento con análogos de la somatostatina, así como su evolución con el tratamiento, según un protocolo establecido con el Servicio de Medicina Nuclear.

Métodos: Se estudió un grupo de dieciocho pacientes con oftalmopatía tiroidea sintomática de dos meses a cuatro años de evolución. Se les inyectó 6 $\mathrm{mCi}$ de ${ }^{111}$ In-octreótido vía endovenosa siendo estudiados mediante SPECT a las cuatro y veinticuatro horas. Cuatro enfermos, todos en fase activa, mostraron captación orbitaria, por lo que fueron tra-

\begin{abstract}
Introduction: Graves' disease, which has an autoimmune basis, is associated with the infiltration of activated lymphocytes into the retrobulbar tissues of the eye. These activated lymphocytes express somatostatin receptors on their surface which mediate the inflammatory response.

Objective: To evaluate the efficiency of ${ }^{111} \mathrm{In}$ Octreotide scintigraphy in the identification of orbital activity in Graves' disease and the usefulness of treatment with a somatostatin analogue, based on a pre-established protocol within the Nuclear Medicine Department.

Methods: We studied a group of eighteen patients with symptomatic Graves' ophthalmopathy of between two months and four years duration. We injected $6 \mathrm{mCi}$ of ${ }^{111} \mathrm{In}$-Octreotide parenterally, and performed scintigraphy four and twenty-four hours later. Four patients, all in the active phase, showed orbital somatostatin receptors, for which they were treated with lanreotide $60 \mathrm{mg}$ every fifteen days for three months. Patients were reviewed after 3
\end{abstract}

\footnotetext{
Recibido: 16/3/06. Aceptado: 13/2/07.

Hospital Universitario San Juan de Alicante. Alicante. España.

1 Licenciado en Medicina.

2 Doctor en Medicina.

Comunicación presentada en el LXXXI Congreso de la S.E.O. (Zaragoza 2005).

Correspondencia:

Fernando Aguirre Balsalobre

Servicio de Oftalmología

Hospital Universitario San Juan de Alicante

Ctra. Nnal. 332 Alicante-Valencia, s/n

Apartado de correos 41

03550 San Juan de Alicante (Alicante)

España

E-mail: faguirr88@hotmail.com
} 
tados con lanreotida $60 \mathrm{mg}$ cada quince días. Se controló a estos pacientes a los tres meses con un nuevo estudio clínico-gammagráfico.

Resultados: En nuestra serie, de los cuatro pacientes que presentaron receptores, todos ellos con evolución menor de dieciséis meses, tres respondieron bien al tratamiento según la valoración posterior.

Conclusión: La gammagrafía con octreótido es un procedimiento útil que diferencia las fases activas, subsidiarias de tratamiento inmunosupresor, en pacientes con oftalmopatía tiroidea. El tratamiento con análogos de la somatostatina fue eficaz en nuestra serie.

Palabras clave: Octreótido, gammagrafía, octreoscan, oftalmopatía de Graves, lanreotida, agentes inmunosupresores, análogos de somatostatina. months treatment with a further scintigraphy and clinical study.

Results: In our study all four treated patients had less than sixteen months of ophthalmopathy, and three responded well to the treatment.

Conclusion: Scintigraphy with Octreotide is a useful procedure allowing identification of patients in the active phase of the ophthalmopathy who are likely to respond well to treatment with somatostatin analogues (Arch Soc Esp Oftalmol 2007; 82: 133-140).

Key words: Octreotide, scintigraphy, octreoscan, Graves' ophthalmopathy, lanreotide, immunosuppressive agents, somatostatin analogues.

\section{INTRODUCCIÓN}

La orbitopatía tiroidea (OT) es una enfermedad de gravedad muy variable tanto clínica como estéticamente que afecta fundamentalmente a mujeres de mediana edad. Están descritas multitud de enfermedades tiroideas que pueden producir alteraciones en estructuras oculares, pero la enfermedad de Graves Basedow es la tiroidopatía con la que más a menudo se relaciona (90\%); aunque tan sólo un 10-12\% de estos pacientes la padecen (1).

La OT consta de dos fases: la fase de inflamación activa, que dura entre 6 y 18 meses de media, y la fase de inactividad, en la que desaparece la inflamación (fase no activa). La fase activa se manifiesta por daño inflamatorio que produce diferentes síntomas como quemosis conjuntival, queratoconjuntivitis, retracción palpebral, movimientos oculares anómalos y diplopías transitorias, proptosis, hiperpresión ocular y neuropatía óptica. Esta inflamación puede, en algunos casos, desembocar en una fibrosis muscular irreversible (2).

Aún no se conocen los mecanismos fundamentales del desarrollo de la orbitopatía, es posible que los mismos auto-anticuerpos antitiroideos agredan a ciertas estirpes celulares presentes en la órbita. Lo que sí está demostrado es la presencia en la fase activa de una infiltración linfocitaria en los tejidos blandos oculares que se acompaña de un estímulo mitótico y madurativo de adipocitos y fibroblastos (3). Los músculos extraoculares aumentan su volumen a expensas de su componente conectivo (1), lo que puede producir alteraciones motoras y restricciones más adelante.

Desde hace unos años se conoce la expresión de receptores de somatostatina por parte de los linfocitos presentes en ese tejido retrobulbar afectado, pero sólo durante la fase activa de la enfermedad (4). La presencia o no de estos receptores puede ser estudiada mediante pruebas objetivas que utilizan la radiación emitida por fármacos análogos de la somatostatina, como el octreótido, unidos a moléculas radioactivas (3). Esto nos ayudaría a diferenciar las OT activas, ya que clínicamente a menudo es difícil (4), y por lo tanto indicar más adecuadamente los tratamientos, que son diferentes a los utilizados en la fase de fibrosis $(3,5,6)$.

Tradicionalmente el manejo de la fase activa se basa en la minimización de la inflamación y el edema mediante inmunosupresores, con clara predominancia de los antiinflamatorios esteroideos, reservándose la radioterapia y la cirugía. Ésta última, en este periodo, sólo se utiliza para complicaciones graves que obliguen a una descompresión retrorbitaria urgente (1). Cuanto mayor sea la severidad de la OT, mejor será la respuesta al tratamiento inmunosupresor (7).

Recientemente se han incluido a los análogos de la somatostatina como una opción para manejar a estos pacientes, basándose en su inhibición de la proliferación y activación de los linfocitos $(6,7)$, pudiendo incluso ser, según algunos autores, una alternativa a los glucocorticoides (4).

En la fase de secuelas sólo la cirugía puede mejorar la calidad de vida. Las manifestaciones más 
importantes son las diplopías ya establecidas y que requieren en muchas ocasiones tratamiento quirúrgico sobre la musculatura ocular extrínseca, así como las retracciones palpebrales que se pueden corregir con «müllerectomías», debilitamientos musculares, o con «espaciadores palpebrales», según los casos.

El manejo adecuado de los síntomas relacionados con problemas de la lubricación ocular debe estar siempre en consideración. Normalmente las lágrimas artificiales proporcionan un alivio moderado y suele ser suficiente en la mayoría de los pacientes (1).

Nuestro objetivo en este trabajo es valorar la efectividad de la SPECT (acrónimo de «Single Photon Emission Computed Tomography») con In ${ }^{11}$ octreótido para identificar la actividad orbitaria en pacientes con la enfermedad de Graves y valorar los candidatos a ser tratados con inmunosupresores, así como la respuesta en control evolutivo a los tres meses de tratamiento con análogos de la somatostatina.

\section{SUJETOS, MATERIAL Y MÉTODO}

Dieciocho pacientes afectos de OT, catorce de ellos mujeres, fueron sometidos a una gammagrafía con receptores de somatostatina para valorar la presencia o no de actividad orbitaria. Todos ellos habían sido diagnosticados previamente de enfermedad de Graves en un rango de tiempo de entre 2 y 45 meses (mediana: 27 meses). La media de edad resultó ser de 34,5 años. A algunos se les presuponía en estado inactivo por el tiempo de evolución, pero si presentaban algún síntoma ocular, tras ser informados y aceptar, se les realizaba la gammagrafía y, de este modo, se valoraba algún grado de actividad y la especificidad.

A cada paciente se le administró una inyección de $6 \mathrm{mCi}$ de $\mathrm{In}^{111}$-octreótido intravenoso, realizando imágenes planares y estudio tomográfico mediante SPECT a las cuatro y veinticuatro horas (fig. 1). Estas imágenes fueron valoradas por los facultativos especialistas de Medicina Nuclear quienes decidieron la presencia o ausencia de actividad a nivel orbitario utilizando un método cualitativo de contraste, comparando la captación orbitaria respecto a la de fondo (tejido óseo occipital) (fig. 2).

Cuatro pacientes mostraron captación del radiotrazador, de forma bilateral aunque asimétrico, todos en fase activa clínicamente.

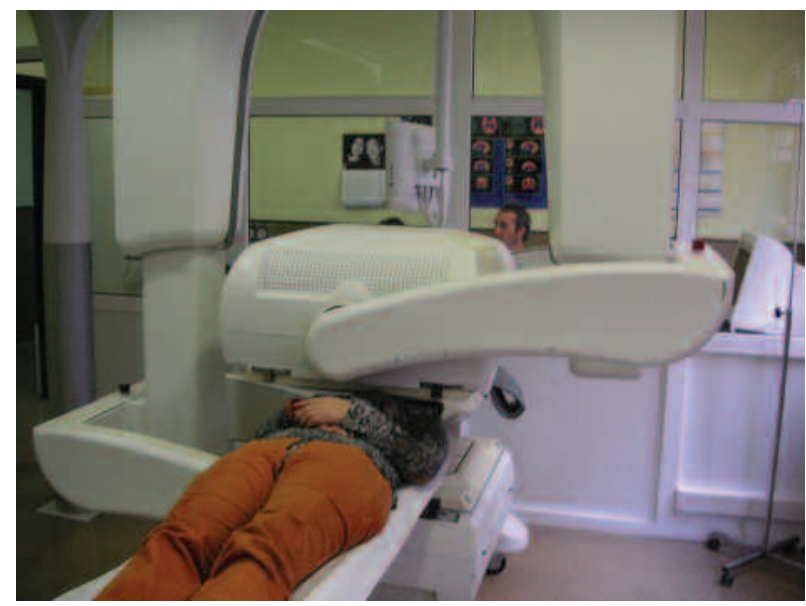

Fig. 1: Paciente siendo sometida a una gammagrafía con In ${ }^{111}$-octreótido.
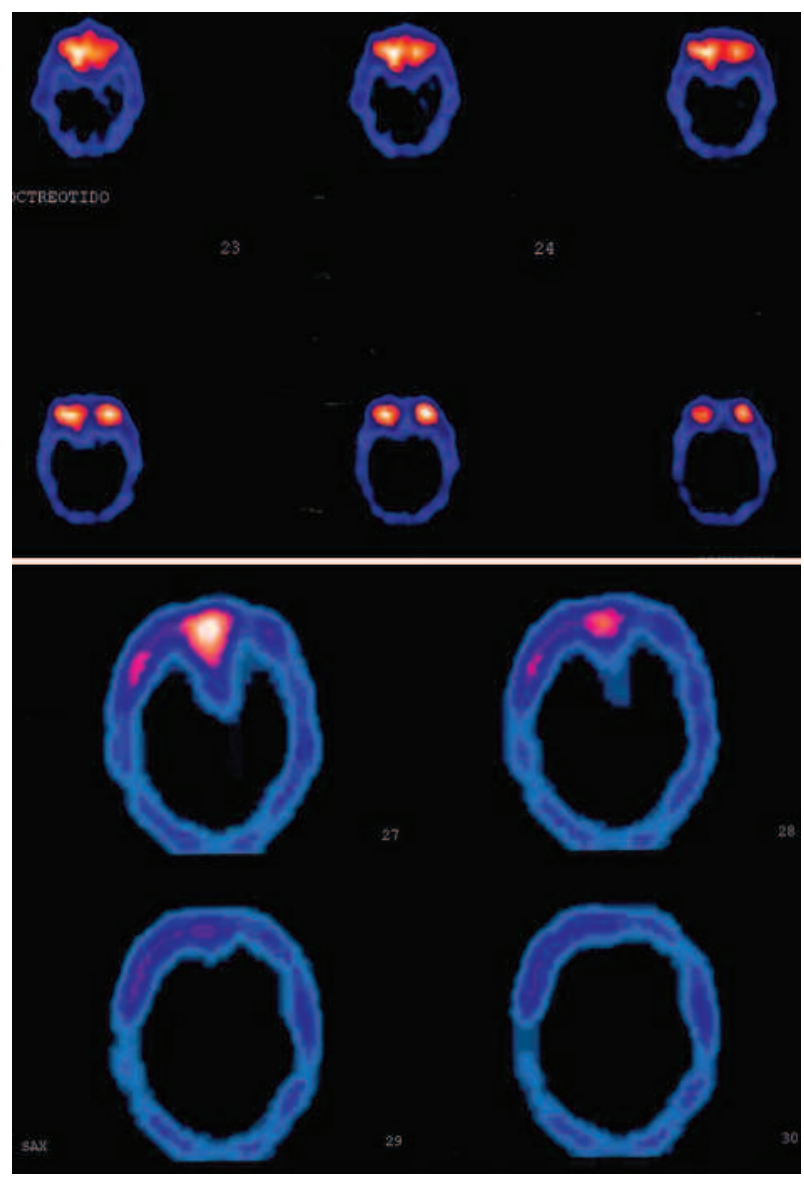

Fig. 2: Arriba: SPECT con receptores (positivo). Abajo: SPECT negativo. 
Los catorce pacientes que se consideraron negativos fueron reevaluados clínicamente pasados de 2 a 5 meses, sin tratamiento inmunosupresor alguno por considerarse que gammagráficamente se encontraban ya en la segunda fase, y no responderían a ningún tratamiento. De ellos, tres pacientes habían sido catalogados como activos clínicamente (dos grado leve de 22 y 25 meses de evolución, y uno grado moderado de 13 meses de evolución). Los once restantes presentaban una puntuación de actividad clínica (PAC) menor de dos y habían sido catalogados como inactivos, con un tiempo de evolución de entre 20 y 45 meses.

El paciente 1 era un varón fumador de 44 años que acudió al Servicio de Urgencias por una queratoconjuntivitis bilateral importante que no respondía a los tratamientos habituales, con estudio microbiológico negativo. En una Interconsulta a Endocrinología se le diagnosticó la alteración tiroidea. Presentaba hipertonía ocular: ojo derecho (OD): 32 $\mathrm{mmHg}$, y 28 en el ojo izquierdo (OI); exoftalmos de $25 \mathrm{~mm}$ en el OD y 24 en el OI; y retracción palpebral de $3 \mathrm{~mm}$ en ambos párpados superiores con 2 mm en párpado inferior del OD y 1 en el OI. Los movimientos oculares externos (MOE) y el resto de exploración resultaron dentro de la normalidad. Según la clasificación de severidad NOSPECS se consideró grado moderado. Se trató a los 2 meses del inicio de los síntomas.

El paciente 2 era una mujer de 54 años que acudió a consultas de Oftalmología por dolor en el OI y diplopía transitoria. En el estudio biomicroscópico se evidenciaba una queratoconjuntivitis bilateral leve y valores de presión intraocular de $20 \mathrm{mmHg}$ en cada ojo. No presentaba exoftalmos ni retracción palpebral. A la exploración de los MOE presentaba un déficit en la supraducción en el OI. Al igual que el paciente 1, en una Interconsulta al Servicio de Endocrinología se le diagnosticó la alteración tiroidea. Se consideró fase activa grado moderado, y el tratamiento comenzó a los 15 meses del inicio de los síntomas.

El paciente 3 era una mujer de 30 años diagnosticada de Enfermedad de Graves 6 meses antes, que fue remitida desde el Servicio de Endocrinología por alteraciones oculares. Presentaba una queratoconjuntivitis bilateral leve con unas presiones intraoculares de $18 \mathrm{mmHg}$ ambos ojos, un exoftalmos, que era de $25 \mathrm{~mm}$ en el OD y 23 en el OI, y retracción palpebral bilateral: OD con $2 \mathrm{~mm}$ de elevación del párpado superior y 1 del inferior y en el OI 1 mm en ambos párpados. Los MOE y el resto de la exploración eran normales. Su severidad era levemoderada y fue tratada a los 4 meses desde el comienzo de los síntomas oculares, en fase activa.

El paciente 4 era una mujer de 27 años que, al igual que la anterior, había sido remitida desde el Servicio de Endocrinología para estudio y tratamiento de sintomatología ocular. Había sido diagnosticada de Enfermedad de Graves 16 meses antes. Presentaba una queratoconjuntivitis bilateral leve con presión ocular de $20 \mathrm{mmHg}$ el ojo derecho y 19 el izquierdo, con exoftalmos de 27 y $24 \mathrm{~mm}$ respectivamente. Retracción palpebral de $3 \mathrm{~mm}$ en párpado superior del OD, y 2,5 mm en ambos párpados inferiores. MOE y resto de exploración normal. Fue considerada activa grado moderado, comenzando el tratamiento a los 10 meses de inicio de los síntomas.

Estos cuatro pacientes fueron diagnosticados de enfermedad de Graves y OT, necesitaron control de la función tiroidea por el Servicio de Endocrinología, y se les trató la orbitopatía con lanreotida 60 mg (Somatulina Autoge ${ }^{\circledR}$; Pisen Pharma, S. A.; Barcelona, España), análogo de la somatostatina de larga vida media, en inyección intramuscular (alternando glúteo) cada quince días durante tres meses. Pasado este tiempo, se les sometió de nuevo a un SPECT orbitario para estudiar cambios en la actividad orbitaria, y además se les reevaluó clínicamente para comprobar concordancia clínico-gammagráfica.

\section{RESULTADOS}

Todas las órbitas que habían sido clasificadas como positivas en el primer SPECT captaron de un modo significativamente menos intenso en el segundo, tras el tratamiento con análogos de la somatostatina. Además la sintomatología se redujo considerablemente en los pacientes 1, 3 y 4 .

Ninguno de los catorce pacientes que resultaron ser negativos mostró cambios clínicos significativos, respecto a su estado anterior, en la reevaluación.

El paciente 1 (grado moderado) ya no refería síntomas de superficie ocular en pocas semanas tras el comienzo del tratamiento, y únicamente necesitó lubricación posteriormente. Se comprobó una reducción del exoftalmos de dos milímetros en cada ojo (hasta 23 y $22 \mathrm{~mm}$ OD y OI respectivamente) y 
de las retracciones palpebrales hasta tan sólo un milímetro en ambos párpados superiores. Hubo una clara mejora gammagráfica (fig. 3).

Sin embargo, la presión intraocular no se logró controlar con tratamiento médico tópico, presentando $30 \mathrm{mmHg}$ en el OD y 36 en el OI; este paciente finalmente requirió solución quirúrgica en el OI, mientras que el derecho alcanzó con tratamiento médico máximo cifras de $16 \mathrm{mmHg}$ a las tres semanas de la evaluación postratamiento, concomitante a la intervención del otro ojo, lo que supone casi cuatro meses en tratamiento con Somatulina Autogel ${ }^{\circledR}$.

La paciente 2 (gravedad moderada) no mejoró clínicamente aunque sí gammagráficamente. Sólo refería mejora del dolor, pero la sintomatología restante, la diplopía, había empeorado; así fue constatado por el Test de Lancaster, que mostró, además, alteraciones en el recto inferior del OD (fig. 4).
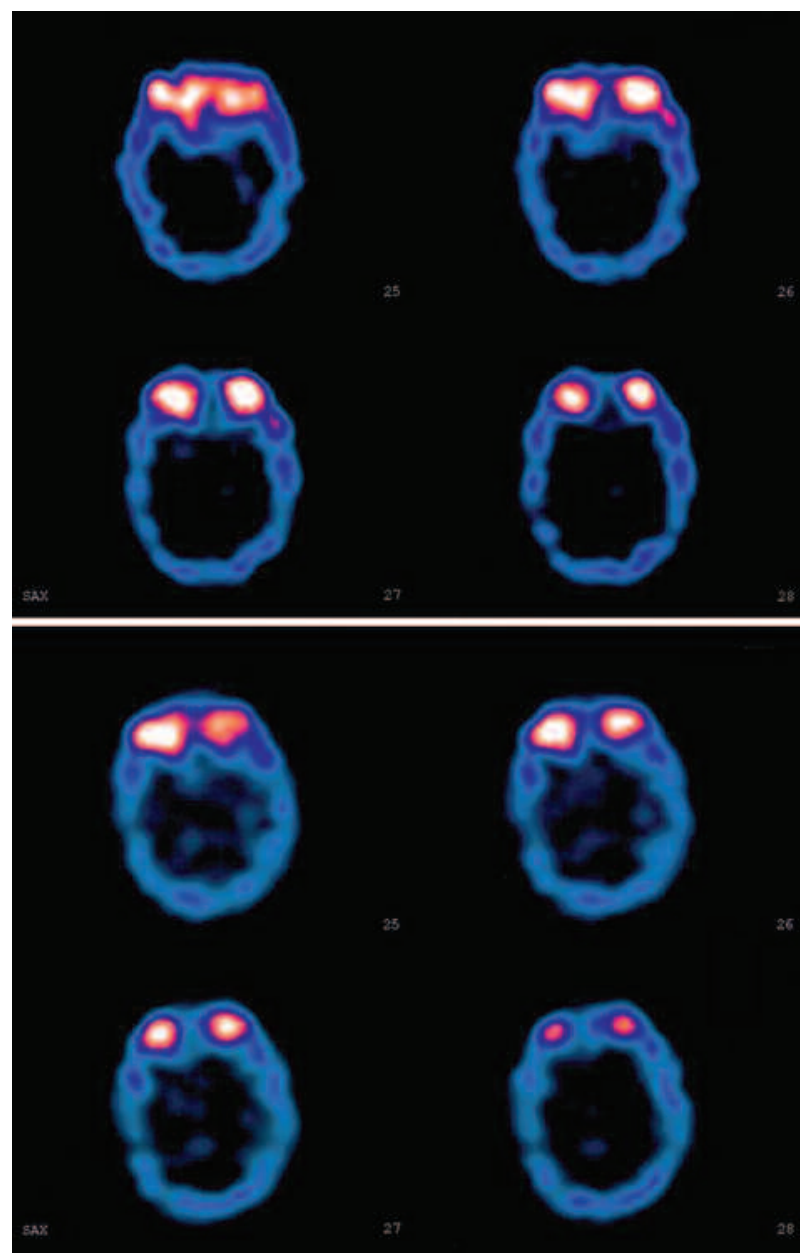

Fig. 3: SPECTs de paciente 1. Superior: pretratamiento. Inferior: postratamiento.
Además la presión intraocular tuvo que ser controlada con análogos de las prostaglandinas tópicas. Como seguía presentando receptores para la somatostatina según el segundo SPECT, se le administraron unas nuevas dosis de lanreotida; después de la cuarta mejoró la clínica y se normalizó la presión intraocular. Tras este segundo ciclo se continuó con un tratamiento corticoideo que se suspendió a los cuatro meses ya que no aportó mejora.

La paciente 3 (leve moderado) mejoró del exoftalmos, disminuyó $2 \mathrm{~mm}$ en el OD y 1 en el OI, y de la retracción palpebral, mostrando superiormente 2 $\mathrm{mm}$ de esclera en OD y $1 \mathrm{~mm}$ inferiormente en este mismo ojo. En el OI no se apreciaban retracciones palpebrales. Resto de exploración sin cambios.

La paciente 4 (grado moderado) mejoró de la queratoconjuntivitis, necesitando únicamente lágrimas artificiales de mantenimiento. El exoftalmos se redujo $1 \mathrm{~mm}$ en ambos ojos. Las presiones se mantuvieron en $18 \mathrm{mmHg}$ el OD y 19 el OI. La retracción palpebral superior del OD disminuyó $2 \mathrm{~mm}$. Como en los demás, el SPECT mostró mejora (fig. 5).

\section{DISCUSIÓN}

De los dieciocho pacientes estudiados, siete se presuponían en fase activa según la actividad clínica, cuatro de ellos grado moderado, uno leve-moderado, y dos leves, considerando a los otros once inactivos. Los cuatro pacientes positivos en el SPECT ya estaban considerados como activos según la clínica, tres de gravedad moderada y uno leve-moderado. Los otros tres pacientes activos clínicamente pero negativos en el SPECT, dos leves y un moderado (22, 25 y 13 meses de evolución), no mostraron cambios en la reevaluación a los tres meses, en todo caso mejoraron, sin tratamiento inmunosupresor.

Cabe destacar que todos los pacientes con más de 15 meses de evolución mostraron negatividad en el SPECT, siendo los pacientes de menor tiempo de evolución positivos (2, 4, y 10 meses). Por ello pasado ese tiempo parece que no es tan útil realizar el SPECT.

Por todo esto pensamos que el SPECT con análogos de la somatostatina marcados radiactivamente ha sido útil para indicar tratamiento médico a los pacientes afectos de OT debida a Enfermedad de Graves, gracias a que ayuda a diferenciar la fase activa de la no activa de esta patología $(2,5,6)$. 

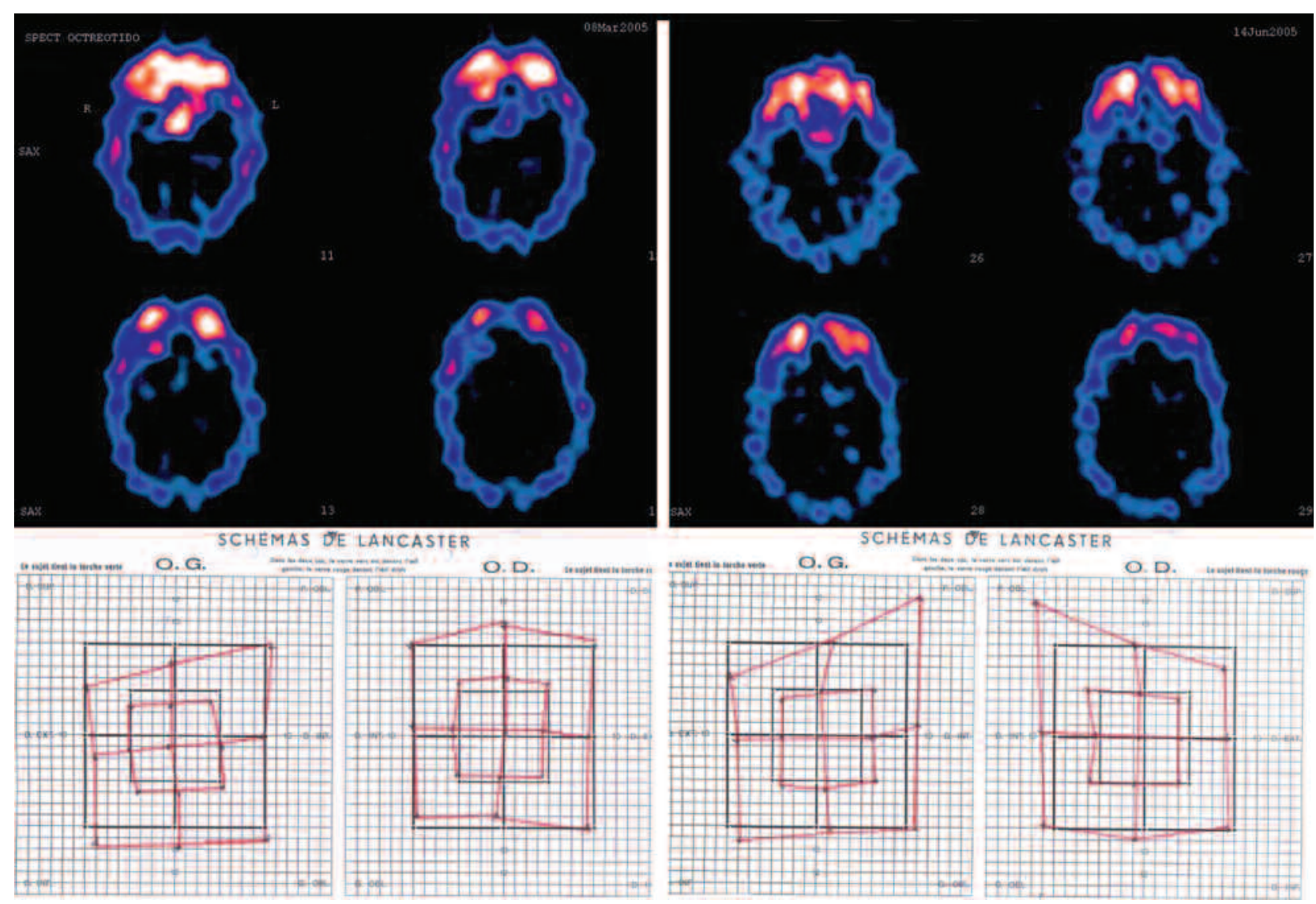

Fig. 4: Paciente 2. Izquierda: SPECT y Lancaster pretratamiento. Derecha: SPECT y Lancaster postratamiento. Observamos que no hay concordancia clínico- gammagráfica; se comprueba menor captación pero más diplopía.

Los pacientes tratados con lanreotida demostraron una menor captación orbitaria y, por lo tanto, una menor actividad inflamatoria ocular, a los tres meses de tratamiento con inmunosupresores. No obstante, en uno de los cuatro pacientes esta menor captación no se relacionó con una mejora clínica significativa.

En nuestra serie de casos, tras el tratamiento, el exoftalmos y la retracción palpebral mejoraron parcialmente en todos los pacientes que los presentaban. En cambio, la miopatía no se alteró clínicamente, y la presión intraocular tampoco mostró cambios significativos. La agudeza visual no presentó cambios en ningún paciente. Las manifestaciones relacionadas con la superficie ocular es lo que más mejoró y más rápido, siendo necesario únicamente tratamiento de mantenimiento con lágrimas artificiales.

Llama la atención, y por lo tanto es digno de mención, la casi ausencia de efectos secundarios (diarrea en la paciente 2).
Dadas las características del estudio, no podemos comparar la efectividad de la lanreotida con los glucocorticoides. Sólo podemos comentar que la paciente 2 no mejoró con ningún tratamiento; o ya estaba en fase inactiva, lo más probable dado el tiempo de evolución, o simplemente no respondió.

No obstante, existen ensayos clínicos que no han demostrado la efectividad de los análogos de la somatostatina, ni ventajas sobre lo glucocorticoides, ni correlación entre el SPECT y la efectividad del tratamiento $(9,10)$. En cambio, la actividad clínica sí que está consensuado que prediga la respuesta a los inmunosupresores (8).

El SPECT con octreótido es una prueba de imagen de realización sencilla y poco invasivo que en nuestra experiencia, y en la de otros autores $(2,3)$, es útil para detectar las fases activas de la OT, susceptibles de tratamiento médico inmunosupresor, así como para predecir la respuesta a análogos de la somatostatina en pacientes con enfermedad de Gra- 

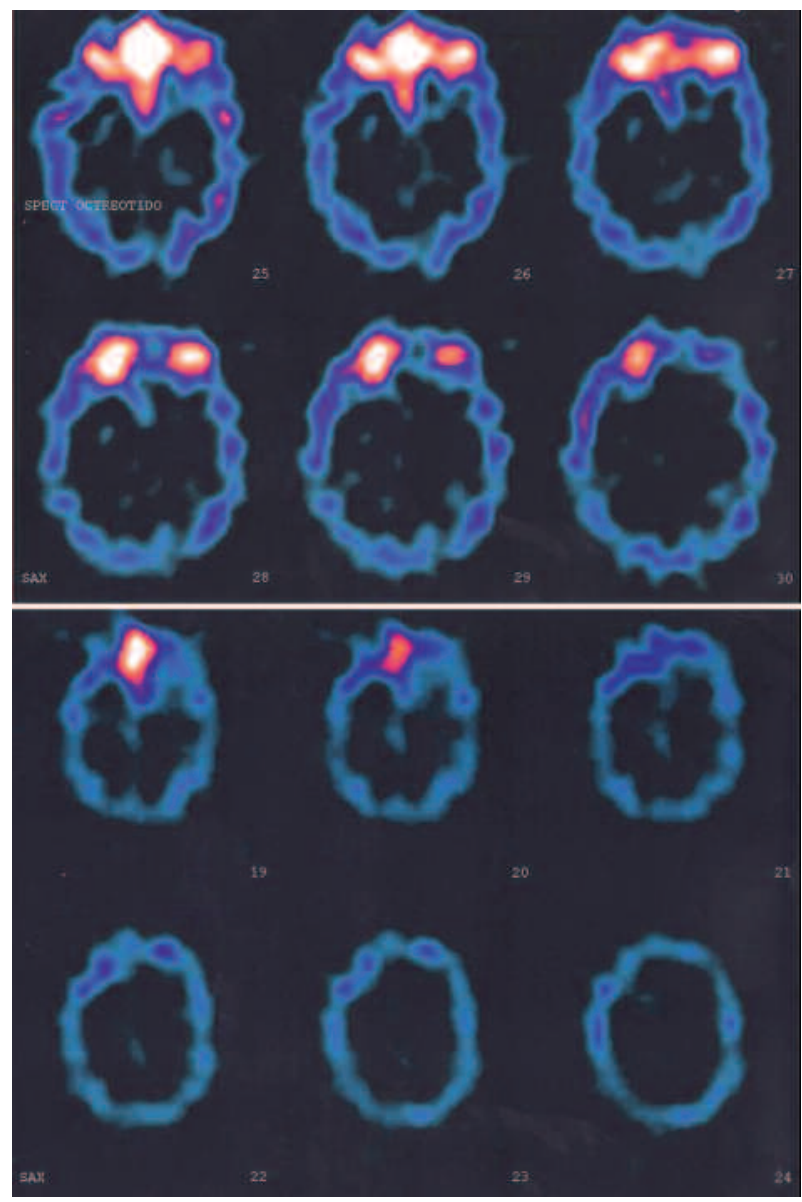

Fig. 5: SPECTs de paciente 4. Superior: pretratamiento. Inferior: postratamiento.

ves. También podría ser un indicador de respuesta a los corticosteroides (5).

Los resultados del tratamiento parece que dependen en gran medida de la selección de los pacientes (menor de once meses en nuestra serie de casos). Es importante que éstos se encuentren en la primera fase de inflamación activa, y una manera objetiva de considerarlo puede ser el SPECT.
Nuevas revisiones y con una serie de casos más extensa obtendremos más datos que nos ayudarán a confirmar, o no, estos primeros resultados.

\section{BIBLIOGRAFÍA}

1. Pérez Moreiras J, Coloma Bockos JE, Prada Sánchez MC. Orbitopatía tiroidea (fisiopatología, diagnóstico y tratamiento). Arch Soc Esp Oftalmol 2003; 78: 407-431.

2. Krassas GE, Kahaly GJ. The role of octreoscan in thyroid eye disease. Eur J Endocrinol 1999; 140: 373-375.

3. Pasquali D, Notaro A, Bonavolonta G, Vassallo P, Bellastella A, Sinisi AA. Somatostatin receptor genes are expressed in lymphocytes from retroorbital tissues in Graves' disease. J Clin Endocrinol Metab 2002; 87: 5125-5129.

4. Krassas GE, Heufelder AE. Immunosuppressive therapy in patiens with thyroid eye disease: an overview of current concepts. Eur J Endocrinol 2001; 144: 311-318.

5. Colao A, Lastoria S, Ferone D, Pivonello R, Macchia PE, Vassallo $P$, et al. Orbital scintigraphy with [ ${ }^{111}$ In-diethylenetriamine/pentacetic acid-D-phe1]-octreotide predicts the clinical response to corticosteroid therapy in patiens with Graves' ophtalmopathy. J Clin Endocrinol Metab 1998; 83: 3790-3794.

6. Krassas GE, Doumas A, Kaltsas T, Halkias A, Pontikides $N$. Somatostatin receptor scintigraphy before and after treatment with somatostatin analogues in patiens with thyroid eye disease. Thyroid 1999; 9: 47-52.

7. Savastano S, Pivonello R, Acampa W, Salvatore M, Lombardi $G$, Colao A, et al. Recombinant thyrotropin-induced orbital uptake of $\left[^{111}\right.$ In-diethylenetriamine-pentacetic acidD-Phe1] octreotide in a patient with inactive Graves'ophtalmopathy. J Clin Endocrinol Metab 2005; 90: 2440-2444.

8. Belzunce-Manterola A, García-Gómez P, Casellas-Bravo M, Heras-Mulero H, Moreno-Montañés J. Oftalmopatía tiroidea: determinación de parámetros de actividad clínica de la oftalmopatía tiroidea como factor de pronóstico de respuesta al tratamiento inmunosupresor. Arch Soc Esp Oftalmol 2005; 80: 705-712.

9. Wemeau JL, Caron P, Beckers A, Rohmer V, Orgiazzi J, Borson-Chazot $M$, et al. Octreotide (long-acting release formulation) treatment in patiens with grave's orbitopathy: clinical results of a four-month, randomized, placebo-controlled, double-blind study. J Clin Endocrinol Metab 2005; 90: 841-848.

10. Dickinson A, Vaidya B, Miller M, Coulthard A, Perros P, Baister E, et al. Double-blind, placebo-controlled trial of octreotide long-acting repeatable (LAR) in thyroid-associated ophthalmopathy. J Clin Endocrinol Metab 2004; 89: 5910-5915. 